\title{
La naturaleza histórica de la irreductibilidad en los paradigmas científicos*
}

The Historical Nature of Irreducibility in Scientific Paradigms

José Alberto Escalante Rodríguez ${ }^{\dagger}$

\begin{abstract}
Resumen
La propuesta filosófica de T. S. Kuhn se distingue por la introducción del análisis de la ciencia desde la perspectiva de su constitución histórica, asumiendo a la historicidad como el atributo "interno" del desarrollo científico, es decir, como su estructura. Dentro de esta concepción, Kuhn ve el cambio como un proceso y no como un momento espontáneo o aislado, enarbolando así precisamente un enfoque distintivo de la ciencia cimentado en el análisis de la formación del conocimiento y no así centralmente desde el de su aplicación, formulación y/o adquisición. Esto le permitirá a Kuhn, concebir a la ciencia como un desarrollo histórico antes que, como una práctica o conjunto de prácticas, antes que como un modo de razonar o pensar determinados, lo cual, se haya estrechamente vinculado a la recepción por su parte, de un posicionamiento ontológico en torno a la finalidad y la limitación.
\end{abstract}

Palabras clave: historicidad - cambio - Kuhn - ciencia - interno - ontología - proceso

\begin{abstract}
The philosophical proposal of T. S. Kuhn is distinguished by the introduction of the analysis of science from the perspective of its historical constitution, assuming historicity as the "internal" attribute of scientific development as its structure. Within this conception, Kuhn sees "change" as a process and not as a spontaneous or isolated moment, raising precisely a distinctive approach of science grounded in the analysis of knowledge formation and not centrally from the point of view of its application, formulation and/or acquisition. This will allow Kuhn to conceive science as a historical development rather than as a practice or set of practices, rather than as a way of reasoning or thinking, which, has been closely linked to his reception of an ontological position about the finality and limitation notions.
\end{abstract}

Keywords: historicity - change - Kuhn - science - internal - ontology - process

\footnotetext{
* Recibido: 14 de abril de 2018. Aceptado con revisiones: 9 de agosto de 2018.

† Universidad Pedagógica Nacional-Hidalgo, México. Para contactar al autor, por favor, escribir a: alberscalan@gmail.com. Metatheoria 10(1)(2019): 13-25. ISSN 1853-2322. eISSN 1853-2330.

(C) Editorial de la Universidad Nacional de Tres de Febrero.

(C) Editorial de la Universidad Nacional de Quilmes.

Publicado en la República Argentina.
} 


\section{La naturaleza histórica del cambio}

La noción kuhniana de estructura refiere al sentido histórico de lo que es "interno", es decir, a la constitución del saber científico en una circularidad dentro de la cual confluyen el pasado y el presente. Así, esta estructura representa propiamente "lo interno", aquello en lo que radica la naturaleza interpretativa de los paradigmas. La noción que describe apropiadamente este fenómeno es la de historicidad, en tanto esta acuña a la "conexión estructural viva" ${ }^{1}$ que expresa el desarrollo, en palabras de Kuhn, "tensional" que se establece con un "origen".

Es posible apreciar en la lectura de Foucault sobre Nietzsche una concordancia explícita con respecto a esta consideración sobre "lo histórico" y más aún, con respecto a la atención con la que debemos hacer eco de la noción de origen para el contexto citado. Nos referimos puntualmente a la distinción que Foucault establece entre Entstehung o Herkunft y Ursprung:

Herkunft [...] es la fuente, la procedencia: es la vieja pertenencia a un grupo - el de sangre, el de tradición,
el que se establece entre aquellos de la misma altura o de la misma bajeza [...] Entstehung designa más bien
la emergencia, el punto de surgimiento [...]. La emergencia se produce siempre en un determinado estado
de fuerzas. El análisis de la Entstehung debe mostrar el juego, la manera como luchan unas contra otras, o
el combate que realizan contra las circunstancias adversas, o aún más, la tentativa que hacen -dividiéndose
entre ellas mismas- para escapar a la degeneración y revigorizarse a partir de su propio debilitamiento.
(Foucault 1979, p. 12)

Desde Nietzsche, Foucault privilegia el sentido histórico de estas formas de concebir la historicidad frente a la concepción lineal que impone "la búsqueda del origen" como un tipo de pretensión historicista que implica un compromiso metafísico con "lo dado".

¿Por qué Nietzsche genealogista rechaza, al menos en ciertas ocasiones, la búsqueda del origen (Ursprung)? Porque en primer lugar [...] se esfuerza por recoger allí la esencia exacta de la cosa, su más pura posibilidad, su identidad cuidadosamente replegada sobre sí misma, su forma móvil y anterior a todo aquello que es externo, accidental y sucesivo. Buscar un tal origen, es intentar encontrar "lo que estaba ya dado", lo "aquello mismo" de una imagen exactamente adecuada a sí; es tener por adventicias todas las peripecias que han podido tener lugar, todas las trampas y todos los disfraces. Es intentar levantar las máscaras, para desvelar finalmente una primera identidad. (Foucault 1979, p. 9)

En la convergencia que podemos hallar entre la noción histórica de desde, que Kuhn usa para describir el "desarrollo" de la ciencia como un proceso "emergente", ${ }^{2}$ con lo que hemos venido reconociendo aquí por historicidad, lo importante a señalar parece referir al estatus ontológico o estructural con el que a través de la noción de desde se asume al pasado. En este sentido, el pasado no resulta en ningún momento identificado como una situación determinada y mucho menos como un "hecho" o un dato, sino como un proceso de transformación; no como lo dado, sino como lo recibido, o en palabras de Kuhn, como "lo reunido con dificultad" (Kuhn 1971, p. 196). De ahí, que el propio Kuhn haga hincapié en que su análisis histórico se dirige al cambio de creencias y no las creencias en sí mismas:

[e]l problema del historiador no consiste en entender porqué la gente sostiene las creencias que tiene, sino porqué eligen cambiarlas, porqué tiene lugar el cambio creciente [...] Para el filósofo de adopta la perspectiva histórica, el problema es el mismo: comprender los cambios de creencia a través de pequeños

\footnotetext{
${ }^{1}$ A grandes rasgos, este concepto como pretendemos aquí usarlo designa el sentido que toma la relación o el nexo interior de un principio con un fin, esto es, como un entre-productivo (Ricoeur 2000, pp. 480-482).

2 En Kuhn, no se puede hablar del pasado en los términos formales de una anterioridad tal como lo podría comunicar la idea de la posibilidad aristotélica. El "pasado kuhniano" tiene más bien la apariencia de un desde y no así de un hacia: "El proceso de desarrollo descrito [...] ha sido un proceso de evolución desde los comienzos primitivos, un proceso cuyas etapas sucesivas se caracterizan por una comprensión cada vez más detallada y refinada de la naturaleza. Pero nada de lo que hemos dicho o de lo que digamos hará que sea un proceso de evolución hacia algo" (Kuhn 1971, p. 263). De tal manera, en dichos términos, la finitud particular del pasado se desarrolla como un vínculo con la tradición, radicando ahí la condición histórica de los procesos. Esto quiere decir que el pasado en la filosofía de Kuhn se concibe como procedencia.
} 
incrementos. Cuando en esto contexto surgen preguntas acerca de la racionalidad, la objetividad o la evidencia, estás se dirigen no a las creencias que eran corrientes antes o después del cambio, sino simplemente al cambio mismo. (Kuhn 1971, p. 196)

Por tal razón, el pasado, desde una "perspectiva histórica” con la que emparenta Kuhn, no resulta en algo que podamos ubicar sobre los términos de un antes o después; sus resistencias con respecto a determinar la naturaleza histórica de los descubrimientos a partir de una estructura cronológica nos advierten ya en parte eso, pero la idea de que la "observación y la conceptuación" o el "algo y el qué", se "encuentran inseparablemente unidos" en un episodio de descubrimiento, parece ser la cuestión central al respecto. En este punto es donde el pensamiento histórico de Kuhn cobra un tono de complejidad esencial en las veces de declaraciones tales como "[e]l aire desflogistizado no es todavía oxígeno." (Kuhn 1982, p. 194).

Sin embargo, comprenderemos que para Kuhn, los descubrimientos científicos no son un fenómeno de "la subjetividad", si como nos es posible ver un poco más adelante, cabe desde un posicionamiento hermenéutico, abordar esta noción en oposición a la idea tradicional que se tiene sobre la elaboración de juicios. ${ }^{3}$ Por el contrario, la relación histórica entre el that-what que compone la "forma" de un descubrimiento (Schindler 2015), y que pareciera anunciarse en el fondo del concepto revolucionario de cambio del que se apropia Kuhn, supone la movilidad de la transformación en un sentido muy parecido al que a continuación mienta Gadamer:

Transformación no quiere decir alteración, por ejemplo, una alteración particularmente profunda. Cuando se habla de alteración se piensa siempre que lo que se altera sigue siendo, sin embargo, lo mismo y sigue manteniéndose como tal. Por mucho que una cosa se altere, lo que se altera en ella es una parte de ella. Categorialmente hablando, toda alteración [...] pertenece al ámbito de la cualidad, esto es, al de un accidente de la sustancia. En cambio "transformación" quiere decir que algo se convierte de golpe en otra cosa completamente distinta, y que esta segunda cosa en la que se ha convertido por su transformación es su verdadero ser, frente al cual su ser anterior no era nada. (Gadamer1999, p. 155)

Apegándonos a eso, bien puede valer decir entonces, que el aire "desflogistizado" se transformó en oxígeno. No obstante, esta sentencia comprende muchas particularidades si es tomada en un tono hermenéutico. Consecuentemente hay que considerar que ni para Kuhn ni para Gadamer, el fenómeno de la transformación o conversión (Kuhn 1982, p. 362), se haya supeditado a la voluntad, sino que comprende más bien un tipo de vivencia que si bien no supone un rechazo de la actividad consciente, localiza a esta como un desarrollo dentro de una esfera más amplia que la del querer y proyectar. ${ }^{4}$

Gadamer reconoce esta experiencia como efectual en términos de una "situación" de la que no se tiene "conciencia plena" (Gadamer 1999, p. 372), y que en cierto modo (y por tal motivo) comprende una circularidad. ${ }^{5}$ Esta experiencia hermenéutica refiere fundamentalmente a la consecución de un proceso que

\footnotetext{
3 “'Sabía Priestley lo suficiente cuando identificó el gas como aire nitroso? [...] sabía él o Lavoisier significativamente más cuando cambiaron la identificación por la del aire común?” (Kuhn 1982, p. 194).

4 "Por eso el verdadero querer y planear de los hombres que actúan no es el objeto auténtico de la comprensión histórica. La interpretación psicológica de los individuos aislados no está en condiciones de alcanzar la interpretación del sentido de los acontecimientos históricos mismos. Ni el sujeto que quiere se agota en esta constelación, ni lo que llegó a ser lo fue por la fuerza de su voluntad, por su inteligencia; no es la expresión pura ni completa de su personalidad" (Gadamer 1999, p. 271).

${ }^{5}$ En clara referencia a la caracterización central que tiene el llamado círculo hermenéutico a partir de la fenomenología heideggeriana (ver Gutiérrez 2000), cuestión que defendemos tiene relevancia para comprender el sentido ontológico que guarda la noción de cambio en el pensamiento de Kuhn: "La movilidad de la comprensión a través de los momentos interpretativos constituye un proceso que en la fenomenología hermenéutica de Heidegger es denominado el círculo del comprender y es considerado un rasgo ontológico de la existencia humana. Gadamer en su hermenéutica filosófica, asimila esta noción del círculo del comprender y la hace productiva para la construcción y el desarrollo del conocimiento en las ciencias del espíritu. Una de las tesis del presente escrito sostiene que la circularidad del comprender también es aplicada por Kuhn en su modelo de construcción y desarrollo del conocimiento científico, desde luego, Kuhn nunca manifestó explícitamente tal situación, sin embargo, un análisis detenido permite colegir que la parte sustancial de la circularidad hermenéutica se encuentra reconocida de manera implícita en su propuesta" (Galván 2009, p. 97). Por otra parte, es necesario resaltar aquí, que la noción de circularidad en el contexto de los desarrollos de la hermenéutica, por lo menos, de Heidegger a Gadamer, aduce fundamentalmente un sentido histórico: "el determinar de nuevo el significado de lo investigado es y sigue siendo la única realización auténtica de la tarea histórica. Sin embargo, el significado se encuentra no sólo al final de tal investigación sino también en su comienzo: como elección del tema de investigación, como estímulo del interés del investigador, como obtención de un nuevo planteamiento" (Gadamer 1999, p. 351). En términos de Kuhn, dicha circularidad histórica puede ser explicada del siguiente modo: "[...] sólo las investigaciones cimentadas firmemente en la tradición científica contemporánea tienen la probabilidad de romper esa tradición y de dar lugar a otra nueva. Ésta es la razón de que hable yo de una 'tensión esencial' implícita en la investigación científica. Para hacer su trabajo, el científico debe adquirir toda una variedad
} 
nos lleva a encontrarnos con la tradición que queremos comprender, o de manera más explícita, podemos decir que tiene lugar cuando reconocemos nuestra pertenencia a la tradición. Resuena así, de nuevo, la idea de que para Kuhn lo importante a destacar sobre el desarrollo de la ciencia sea precisamente la naturaleza histórica del cambio de creencia, es decir, el cómo se ha llegado a producir el cambio mismo. Este "acontecimiento" o evento extraordinario es al que hacemos referencia desde una perspectiva hermenéutica a través del concepto de situación, definiendo a este como la consecución de un proceso de cambio, el cual señala a la experiencia que tiene lugar mediante el rompimiento con una formación o proceso. Parece ser entonces, que es esto lo que en esencia habrá que reconocer como la célebre "tensión esencial" kuhniana.

$\mathrm{Al}$ respecto, creemos que el propio Kuhn recurre a la noción de conocimiento tácito con el fin de matizar el sentido "involuntario" de este proceso. El carácter más bien de "anonimato" que involucra a la noción de transformación (Gadamer 1999, pp. 156-157), supone que lo que hay de por medio en tal constituyente, no es un objeto a transformar sino al acontecimiento mismo de la transformación, lo cual exige en esencia concebir al descubrimiento científico como algo en lo que se participa y no propiamente algo "que se hace", en contraste con la agencialidad que se le puede atribuir al descubrimiento desde una perspectiva epistemológica. ${ }^{6}$ Sin embargo, no intentamos decir con esto que la postura de Kuhn sostenga la idea de un determinismo de tipo alguno (o en este caso histórico), que haga parecer a los individuos insertos en un paradigma bajo la suerte de un carácter enajenado, sino que por el contrario, pretendemos sugerir que una de las diferencias "esenciales" a vislumbrar en los planteamientos kuhnianos sobre la producción del conocimiento, con respecto a lo que se propone comúnmente desde la tradición epistemológica clásica, o como pretende decir Kuhn, "a partir de Descartes pero no antes”, radica en la particularidad de la "acción” o "participación" de los individuos para cada caso. Así pues, mientras Kuhn remite esta "participación" o "producción" al ámbito de las creencias recibidas, como el propio Kuhn nos lo señala, por su parte, la tradición epistemológica se decanta por tomar como su objeto principal, la acción sobre "lo dado" (Kuhn 1971, p. 197).

A simple vista, el término "participar" puede sugerir cierta ambigüedad si no se le dota del sentido filosófico que le adscribe su recorrido histórico. Sin embargo, a pesar de que pueden resultar más inmediatas sus referencias metafísicas, ${ }^{7}$ estamos considerando la noción de participación desde el punto de vista de la constitución de la experiencia de los individuos que conforman un paradigma, ${ }^{8}$ en tanto es esta perspectiva, la que nos invita a pensar en la clase de relación que se puede asociar a la dinámica de una comunidad bajo los términos en los que Kuhn concibe su formación al nivel de las creencias compartidas. En este sentido, los científicos se vislumbran partícipes del proyecto de la comunidad mediante la creencia en su proyecto, lo cual, nos posiciona ya frente a un concepto de creencia que no se arropa bajo la carga

de compromisos intelectuales y prácticos. Sin embargo, su aspiración a la fama, en caso de que tenga el talento y la buena suerte para ganarla, puede estar fundada en su capacidad para abandonar esa red de compromisos a favor de otros que él mismo invente" (Kuhn 1982, p. 250).

6 "[Según Anscombe] el signo primitivo de querer es tratar de obtener, lo cual desde luego sólo puede adscribirse a criaturas dotadas de sensación. En consecuencia, no se trata de un simple movimiento ni de avanzar hacia algo, sino que es esto realizado por una criatura de la cual puede decirse que conoce el objeto" (Broncano 2006, p. 9).

7 Como adquiere en la teoría de las ideas de Platón y que ya nos señala Aristóteles como un remanente de la concepción pitagórica de la imitación (Aristóteles 2008, p. 57).

8 Haciendo un símil con las particularidades que Karl Kerényi reconoce en torno a la experiencia de la ritualidad festiva en la antigüedad helénica (Kerényi 1999, p. 43), sugerimos la idea de participación para explicitar la relación que guardan los individuos con un paradigma. En este sentido, no consideramos que este participar supone la práctica dirigida para la consecución de un fin o la producción de un objeto, como tampoco nos referimos con ella a la metafísica asentada en la teoría platónica de las ideas, sino que pretendemos indicar que su concepto se encuentra en la base de la constitución misma de la creencia en un paradigma, lo cual sostenemos que desde una perspectiva kuhniana, demarca fundamentalmente el fenómeno de la pertenencia a tal. Aducimos esto en particular, cuando Kuhn nos señala cosas tales como que, durante los periodos de ciencia normal, los científicos no intentan sino "realizar la promesa" que el mismo paradigma inaugura, más allá de que "la búsqueda de nuevos fenómenos", o que en tal caso, la naturaleza misma de la investigación normal corresponde al desarrollo de la aceptación de un paradigma, entiéndase pues, al desarrollo del compromiso que implica a la pertenencia como un atributo de la comunidad (ver Kuhn 1971, pp. 51-67). 
psicológica de la noción de disposición, ${ }^{9}$ y que por el contrario, se emparenta directamente con la idea de conocimiento heredado, un proceso de formación anclado en el reconocimiento de una autoridad, que por decirlo de algún modo, "organiza" las relaciones al interior de la comunidad. El carácter participativo de los miembros de la comunidad es a su vez un rasgo que podemos ver asociado al sentido de creación (Kerényi 1999, p. 49) de los individuos que participan en ella, y por eso, a la producción conformada con respecto a la "fe" que involucra a un paradigma y así al reconocimiento de un Leitmotiv compartido mediante la educación. Esto, en esencia, parece indicar que, para que un científico se convierta a un paradigma, "tiene" que estar de alguna forma "inmiscuido" o involucrado con él sin a priori "de por medio". Sin embargo, esto no quiere decir que el científico sea aquél individuo capaz de aplicar un paradigma, crear no es aplicar, su relación no es técnica. Esto puede clarificarse si atendemos a los esfuerzos de Kuhn por defender que lo que tiene lugar en el fenómeno del cambio no se parece nada a una elección, del mismo modo en el que tampoco resulta posible elegir una teoría sin antes tener conocimiento "pleno" de las opciones disponibles, es decir, no se puede adoptar un paradigma sin antes haber experimentado sus efectos, o en específico, dentro de la problemática que nos plantea Kuhn, sin antes haberlo aprendido (Kuhn 1982, pp. 363-364).

\section{El sentido ontológico de la historicidad}

Ahora bien, es necesario acotar lo que se está queriendo dar a entender con el término situación en una connotación hermenéutica, toda vez que, al momento, atisbamos que desde la perspectiva histórica que le imprime Kuhn a sus planteamientos sobre el cambio científico, todo proceso o movimiento puede ser tratado hermenéuticamente bajo el concepto mismo de una situación, sin embargo, es necesario tomar ciertas consideraciones al asociar este concepto a la noción de lo interno o estructural. Para esto habrá que rastrear el concepto de situación a partir de la lectura que Gadamer hace de Karl Jaspers, explícitamente, refiriéndonos al texto "Ambiente Espiritual de Nuestro Tiempo" (Gadamer 1999, p. 372). Bajo tal referencia, Gadamer describe a la situación como algo en lo que "se está" y no como algo que se tiene "en frente". Sin embargo, siguiendo el hilo conductor entre ambos pensadores, también tenemos que una situación es algo a lo que se llega, ${ }^{10}$ pero no así en el sentido de un desplazamiento que suponga la condición de un llegar hacia, pues:

[clomprender una tradición requiere sin duda un horizonte histórico. Pero lo que no es verdad es que este horizonte se gane desplazándose a una situación histórica. Por el contrario, uno tiene que tener siempre su horizonte para poder desplazarse a una situación cualquiera. ¿Qué significa en realidad este desplazarse? Evidentemente no algo tan sencillo como "apartar la mirada de sí mismo" [...] uno tiene que traerse a sí mismo hasta esta otra situación. (Gadamer 1999, p. 375)

Bajo este orden de ideas, el término situación no puede confundirse con trasfondo, y en este sentido, lo que se dice por "interno" no debe pasar por la idea de "trasfondo" que desde una perspectiva subjetivista describe a la apropiación consciente del contexto de una época, cosa que por ejemplo suele ocurrir a partir del análisis situacional popperiano, esquema en el que precisamente "lo interno" se asocia a la idea de "trasfondo", concepto que a su vez parece entenderse con referencia a lo oculto:

Popper propone el análisis situacional como un método de comprensión objetiva, el cual consiste en reconstruir la situación problemática en la que se encontraba el científico, o en general cualquier agente cuyas obras requiere comprender. La situación problemática está constituida por el problema al que la teoría intenta responder, y por el trasfondo o marco cognoscitivo, en el que el autor se plantea el problema y busca resolverlo [...]. Es muy importante enfatizar la idea de trasfondo (background), justamente porque define una situación determinada que posibilita plantear ciertos problemas, conjeturar ciertas soluciones y

\footnotetext{
9 En el sentido por ejemplo en el que Popper concibe a las creencias, más precisamente, el origen de las creencias, como una característica psicológica fuera de la competencia de todo tratamiento epistemológico por el hecho de tratarse de un "fenómeno" de sentido común (ver Popper 1962, pp. 19-94, González 2004, pp. 131-144).

${ }^{10}$ Siguiendo a Jaspers, nos estaríamos refiriendo a una situación como a algo que se origina o se gesta, con la premura de las acotaciones que ya hemos señalado para el término origen en el espíritu de este texto (Jaspers 1933, pp. 9-24).
} 
evaluarlas de manera crítica. Esta noción de trasfondo me parece que es sustituible por el concepto de tradición (de primer orden) que Popper desarrolla en otros textos. El trasfondo o las tradiciones específicas constituyen el marco lingüístico y teórico indispensable para comprender las teorías científicas del pasado y evaluar la racionalidad del autor al proponerlas y defenderlas. (Velasco 2004, p. 77)

Tal forma de caracterizar el concepto de "situación", que creemos se aproxima a asumir el pasado como un hecho, nos hace evocar de momento el espíritu crítico de Gadamer frente a todo subjetivismo, cuando este le increpa a Dilthey el pensar "la investigación del pasado histórico como desciframiento y no como experiencia histórica” (Gadamer 1999, p. 303). Resulta ser así, que el pasado, en una perspectiva hermenéutica, no puede suponer una relación de enajenación o de ocultamiento que demarque una distancia que haga suponer a su vez la necesidad de un desciframiento, pues si nos atenemos de nuevo a lo que nos dice Gadamer:

En nuestro comportamiento respecto al pasado que estamos confirmando constantemente, la actitud real no es la de la distancia ni la libertad respecto a lo transmitido. Por el contrario, nos encontramos siempre en tradiciones, y este nuestro estar dentro de ellas no es un comportamiento objetivador, que pensara como extraño o ajeno a lo que dice la tradición; ésta es, siempre más bien algo propio, ejemplar o aborrecible, es un reconocerse en el que para nuestro juicio histórico posterior no se aprecia apenas conocimiento, sino un imperceptible ir transformándose al paso de la misma tradición. (Gadamer 1999, p. 350)

El sentido de "enajenación" con el que Popper considera al pasado como un hecho, queda de manifiesto con respecto a la nula movilidad que le adscribe este a las tradiciones. Así pues, cabe señalar el sentido estático que comunica la idea popperiana de las "tradiciones de primer orden", es decir, "las tradiciones concretas formadas por teorías y mitos en específicos, que transmiten conocimiento sustantivo desarrollado por generaciones pasadas", ${ }^{11}$ pues en la perspectiva popperiana, los prejuicios de una época, el trasfondo, o "la tradición de primer orden", no presentan una movilidad propia, sino que son concebidos como cuerpos estáticos de la misma manera en que para términos concretos es pensada la "meta-tradición” crítica:

Las tradiciones de primer orden son plurales, surgen siempre en contextos históricos específicos, y cambian de manera continua como resultado de la evaluación crítica que de ellas se hace desde la tradición de segundo orden. Este último tipo de tradición es única, invariable y de alcance universal. Las tradiciones específicas de primer orden son pasivas en cuanto que sólo proporcionan el material para la evaluación crítica; en cambio la tradición crítica de segundo orden es activa, en cuanto realiza el escrutinio crítico de las tradiciones y de esta manera fomenta su cambio y progreso. Sin embargo, la tradición crítica no es reflexiva, no se someta a sí misma a crítica, y por ende no cambia en forma progresiva. (Velasco 2004, p. 75)

Así, el esquema de cambio científico de Popper, se concibe a partir de dos estructuras diferenciadas tanto por su función como por su naturaleza; es decir, una estructura "pasiva” y otra "activa" que "no sólo cumplen funciones distintas (fuente y fundamentación de conocimiento), sino que también tienen características muy diferentes" (Velasco 2004, p. 75). Pensar este esquema desde la concepción de ciencia normal y extraordinaria puede resultar atractivo y engañoso a la vez. Buena parte de las críticas que Kuhn vierte en "La lógica del descubrimiento o la psicología de la investigación", se decantan en dar cuenta de este malentendido:

Sir Karl se equivoca al transferir características seleccionadas de la investigación cotidiana a los ocasionales acontecimientos revolucionarios en los cuales el avance científico es más obvio, y al pasar por alto en adelante, a la actividad cotidiana”. En particular, trata de resolver el problema de la elección de teoría

\footnotetext{
${ }^{11}$ La tarea de justificar el conocimiento corresponde a otro tipo de tradición que es más bien una metatradición transhistórica, carente de contenidos cognoscitivos específicos y que consiste en una actitud y metodología críticas. Esta tradición de segundo orden es el racionalismo crítico, que inventaron los filósofos griegos hace más de 2000 años, y aun en nuestros días permanece sin cambios como el rasgo más importante del conocimiento científico. Gracias a esta tradición crítica puede superare la actitud tradicionalista y dogmática y es posible vivir de manera racional en y mediante tradiciones específicas que transformamos continuamente (Velasco 2004, p. 74).
} 
durante las revoluciones conforme a criterios lógicos aplicables totalmente sólo cuando una teoría ya puede darse por sentada. (Kuhn 1982, p. 312)

En la perspectiva de Kuhn, ni la ciencia normal, ni la ciencia extraordinaria refieren a estructuras aisladas, sino a procesos. La idea de una estructura en Kuhn no se vincula a la idea matemática de un conjunto u orden, ni a la epistémica de un sistema, sino que refiere al movimiento interno del proceso de cambio. Dicho de otra manera, desde la posición de Kuhn, al constituirse como tradiciones, los paradigmas no se estructuran desde lo completo y cerrado en sí mismo, ${ }^{12}$ ni por lo tanto a partir de los problemas que se ha "fallado" en resolver, sino desde las posibilidades de ver dentro un determinado dominio de formación (u horizonte). ${ }^{13}$

La naturaleza de la noción kuhniana de estructura, frente a otras manera de concebir lo "interior", refiere precisamente a la idea de algo en formación, pues para Kuhn, la idea de una "interioridad" recoge la temporalidad de un proceso constituido desde el pasado, de tal manera que puede concebirse al pasado como una posibilidad heredada, es decir, en el sentido de la noción de una continuidad entendida como procedencia:

El éxito de un paradigma -ya sea el análisis del movimiento de Aristóteles, los cálculos hechos por Tolomeo de la posición planetaria, la aplicación hecha por Lavoisier de la balanza o la matematización del campo electromagnético de Maxwell- es al principio, en gran parte, una promesa de éxito discernible en ejemplos seleccionados y todavía incompletos. La ciencia normal consiste en la realización de esa promesa (Kuhn 1971, p. 52).

En este sentido, la dinámica que Kuhn considera habida entre una paradigma anterior y uno alternativo, no puede entenderse en términos de alguna dicotomía, tal como ocurre por ejemplo en el modelo pasivoactivo popperiano; por decirlo de otra forma, de acuerdo con Kuhn, esta relación transformativa no se estructura desde la argumentación ni desde el "convenio", por lo que no aduce una naturaleza lógica como tampoco "socio-lógica". ${ }^{14}$ Por el contrario, para Kuhn, tanto la ciencia normal como la ciencia extraordinaria constituyen procesos históricos. De tal modo, la conversión a un paradigma supone la formación de un sensus por parte de la comunidad acerca de su propia finitud y por lo tanto también acerca de su propio desarrollo, es decir, una conciencia de su desde, fundamentalmente de su situación hermenéutica, experiencia que Kuhn describe frecuentemente como "reconocer que algo ha salido mal de una manera que puede tener consecuencias importantes” (Kuhn 1971, p. 197); de ahí por ejemplo, que se diga que el preludio a un descubrimiento no sea un asunto de "ignorancia", sino de toma de conciencia de lo que se sabe y de lo que se cree (Kuhn 1982, p. 258).

Esta "formación de la conciencia" o mejor dicho, del sentido, constituye un punto central en la propuesta gadameriana en tanto la experiencia hermenéutica de la interpretación comprende una iluminación parcial sobre la pertenencia a la tradición ${ }^{15}$, lo cual, esencialmente distingue una especie de darse cuenta de la situación en la que uno se halla, elemento de base en la formación del sentido de pertenencia a la comunidad, y que se le reconoce como parcial, en tanto supone la asunción del porqué se ha llegado a determinada situación. Este planteamiento supone una fundamentación histórica del desarrollo desde el punto de vista de una finitud no impuesta, sino en proceso. La pregunta en cuestión que debe desprenderse de ahí, es entonces la que hace referencia a la afectación del pasado, no en sí, como lo pensaría Popper, la pregunta por el significado del pasado (Popper 2006, p. 471). El viraje se da entonces conforme al

\footnotetext{
${ }^{12}$ En el caso contrario tenemos la postura de Popper cuando dice por ejemplo: "La vida social sólo puede existir si podemos saber y tener la seguridad de que hay cosas y acontecimiento que deben ser así y no pueden ser de otra manera” (Popper 1983, p. 167).

${ }^{13}$ De nueva cuenta, la naturaleza de lo que puede asumirse como "racional" desde la postura kuhniana, encuentra diferencias de peso con respecto a lo que se aduce del mismo modo en términos de Popper: "[...] creamos la nueva teoría con el fin de resolver aquellos problemas que la vieja teoría no podía resolver" (Popper 1983, p. 169).

14 "No debemos interesarnos por los argumentos que de hecho convierten a uno u otro individuo, sino más bien por el tipo de comunidad que siempre, tarde, o temprano, se reforma como un grupo único" (Popper 1983, p. 169).

15 “[...] en esta comprensión habrá siempre algo más que la reconstrucción histórica del 'mundo' pasado al que perteneció la obra. Nuestra comprensión contendrá siempre al mismo tiempo la conciencia de la propia pertenencia a ese mundo. Y con esto se corresponde también la pertenencia de la obra a nuestro propio mundo" (Gadamer 1999, p. 359).
} 
posicionarnos en torno al efecto del pasado o en cuanto al decidir sobre el pasado, es decir, en esta última acepción, de nueva cuenta considerando a la historia como algo que se hace. ${ }^{16}$

Dicho lo anterior, se nos haría difícil considerar el que Kuhn estuviera del lado de aceptar alguna clase de separación analítica entre la ciencia normal y extraordinaria, tal como la perspectiva "racional" de la crítica popperiana podría sugerirlo. ${ }^{17}$ En este sentido, el Kuhn en clave hermenéutica, muestra al fenómeno del cambio como la posibilidad de ver las cosas transformadas, ${ }^{18}$ no como la posibilidad de elegir entre "cosas" distintas (Kuhn 1982, p. 362).

El que Popper plantee "la pregunta por la historia" con respecto a una cuestión sobre el significado de la misma, ya parece distanciarnos de raíz con respecto a la idea que podemos encontrar en Kuhn de que la historia confiere más bien a una relación con el pasado a partir de la cual se estructura el cambio que tiene lugar con respecto a nuestra voluntad y no precisamente al margen de ella, cuestión que parece difícil de asimilar sobre todo si la noción de voluntad como "experiencia de vida" que nos vincula con una comunidad, es confundida con una noción de libertad como poder de decisión. ${ }^{19} \mathrm{Al}$ respecto, cabe decir que es el mismo Popper quien pone en la base de su epistemología un dualismo entre hechos y decisiones (Popper 2006, p. 490), cuestión que en el concierto de la reflexión kuhniana no parece encuadrar si nos remitimos a la forma en la que Kuhn se distancia de realizar una oposición entre objetividad y subjetividad: "Si mis críticos introducen el término "subjetivo" en sentido opuesto a "relativo a juicios" -sugiriendo así que hago de la elección de teoría u asunto indiscutible, un asunto de gusto-, entonces es que han confundido seriamente mi posición” (Kuhn 1982, p. 362).

En este sentido, Kuhn parece no estar pues por entender "lo subjetivo" en términos de una disposición de las opciones que supone una elección, por el contrario, nos atreveríamos a decir que en el matiz en el que en el vocabulario de sus críticos se habla de lo subjetivo, cabría mejor referir al término creencias precisamente ahí donde Kuhn refiere a "los factores idiosincrásicos dependientes de la biografía y la personalidad del sujeto” (Kuhn 1982, p. 354).

En todo caso, por encima del punto de vista en el que la racionalidad de la historia converge en una selección (orden) de momentos o eventos decisivos o por lo menos que competen al ámbito de la decisión $^{20}$, la consideración más fuerte que hace Popper sobre la historia, radica en considerarla precisamente en la antípoda de la toma de decisiones. Por eso, desde su enfoque, el problema de la historia se entiende a través de lo que parece describirse por el carácter determinista de su imposición. Pero la solución que plantea el mismo Popper está del lado de imponerle a esta imposición el atributo de nuestra voluntad, de nueva cuenta, considerando aquello de la "voluntad" en el espectro de algo que se hace y no como algo que acontece. ${ }^{21}$ En cuanto a esto, cobra relevancia considerar la declaración popperiana de que "la historia no tiene sentido pero podemos dárselo". ${ }^{22}$ En tal caso, lo que para Popper le da sentido a la historia son las decisiones correctas, al reafirmar aquella necesidad del carácter crítico que pregona sobre el "aprender de nuestros errores". De esta manera, Popper no concibe a las tradiciones como históricas, sino como "algo que podemos criticar y cambiar" (Popper 1983, p. 168). Así pues, la historia, desde el punto de vista popperiano, vendría a referir únicamente a lo que limita a las tradiciones, es decir, a lo que las constituye como "sistemas de control". ${ }^{23}$

\footnotetext{
16 "Los hechos, ya sean de la naturaleza o de la historia, no pueden decidir por nosotros, no pueden determinar los fines que hemos de elegir. Somos nosotros los que le damos una finalidad y un sentido a la naturaleza y a la historia" (Popper 2006, pp. 489-490).

17 "En particular, trata de resolver el problema de la elección de teoría durante las revoluciones conforme a criterios lógicos aplicables totalmente sólo cuando una teoría ya puede darse por sentada" (Kuhn 1982, p. 312).

${ }^{18}$ En todo caso, partiendo de Gadamer, podríamos incluso decir que la hermenéutica comprende un modo de ver particular y no así propiamente un método (Gadamer 1999, p. 350).

${ }^{19}$ O lo que creemos que reconocería Popper como "la responsabilidad de nuestros actos" (Popper 2006, p. 490).

20 "El historiador popperiano buscar teorías falsables, importantes y audaces, y grandes experimentos cruciales de resultados negativos" (Lakatos 1983, p. 142).

21 "A mi juicio, ese dualismo de hechos y decisiones es fundamental. Los hechos, como tales, carecen de significado, sólo pueden adquirirlo a través de nuestras decisiones" (Popper 2006, p. 490).

22 "Si bien la historia carece de fines, podemos imponérselos, y si bien la historia no tiene significado, nosotros podemos dárselo" (Popper 2006, p. 489).

${ }^{23}$ En estrecha relación con la "institucionalidad" bajo la que Popper concibe a las tradiciones (Popper 2006, p. 170).
} 
Por otro lado, cabe decir que, bajo la perspectiva racionalista-crítica, la única fuente de movilidad de la tradición consiste en un fenómeno psicológico que tiene lugar mediante la imitación (Popper 1983, p. 171). Sin embargo, de nueva cuenta podemos marcar una diferencia clara con respecto a lo que Kuhn entiende por la movilidad de una tradición, pues para el filósofo norteamericano, es el aprendizaje, la pauta de la transmisión del conocimiento: "Hay un largo paso del rechazo de la idiosincrasia psicológica de un individuo al rechazo de los elementos comunes inducidos por la educación y el adiestramiento dentro de la conformación psicológica del miembro titulado de un grupo científico" (Kuhn 1982, p. 315).

Así pues, donde Kuhn ve en la conformación de la tradición un compromiso ontológico, es decir, un vínculo con los referentes del mundo a través de la educación, Popper ve imitación psicológica. Esto nos muestra que desde el racionalismo popperiano, la historia se circunscribe básicamente a la regularidad del comportamiento individual, la cual, fuera del ámbito de la crítica, se supone como un "sin sentido".

Si bien recordamos, Kuhn también observa que la historia no lleva una ruta definida de antemano, no ve en esto una falencia o una limitación del cambio, sino una cualidad intrínseca del mismo. Por el contrario, pareciera ser que en la visión de Popper, es indispensable de nueva cuenta tener un fin, un punto precedentemente definido como requisito para poder dar lugar al cambio. La actitud crítica viene a ser pues en este sentido, aquello que llena el vacío entre los fines y las metas. Bajo esta concepción ahistórica del cambio, la tradición representa a un conjunto ordenado de elementos. Sin embargo, no podemos decir entonces que sea lo mismo pensar la tradición como un "orden" en nuestras creencias o expectativas, que considerar que nuestras creencias, motivos y expectativas se "ordenan" en una tradición.

En igual tono de discordancia, para Kuhn, la justificación y el descubrimiento son procesos constitutivos del desarrollo histórico de la ciencia, lo que quiere decir que la movilidad de esta se produce en confluencia con estos dos factores. Por su parte, para Popper, la justificación del conocimiento científico sigue siendo un problema metodológico, un problema entre lo "verdadero" y su distorsión, es decir, cuyo único nexo viene a concebirse desde la "actitud crítica", que como hemos revisado escuetamente, funge siempre como un elemento externo al desarrollo mismo de la ciencia.

Parece ser entonces que, para una posición racionalista como la descrita, lo que se vuelve motivo del "análisis crítico" son las "ideologías" como fuentes de error y no el vínculo histórico entre las creencias y el mundo. De tal modo, las creencias son tomadas como deformaciones de la realidad una vez que la historia ha hecho su tarea, es decir, que ha llevado a su límite el progreso. Considerar a la tradición como un fenómeno psicológico y a-histórico conviene entonces a considerarla del lado de la ideología:

\footnotetext{
La "falsa conciencia" (falsa desde el punto de vista de su teoría de la racionalidad) crea un problema al historiador falsacionista. Por ejemplo, ipor qué algunos científicos entienden que los experimentos cruciales son positivos y verificadores en lugar de negativos y refutadores? Para solucionar estos problemas el falsacionista Popper elaboró (con más habilidad que cualquiera de sus predecesores) el puente entre el conocimiento objetivo (en su "tercer mundo") y sus distorsionados reflejos en las mentes individuales. (Lakatos 1983, p. 144)
}

Interpretar a la incompletud que acompaña a los paradigmas como error y por lo tanto a las anomalías como "distorsiones", revela la inclinación psicológica aquí señalada (Lakatos 1983, p. 164), pero en el marco de una concepción hermenéutica del cambio, el "no haber visto algo como algo" no puede ser considerado como un error, o tomado como una inconsistencia: "No se cometió ningún error para llegar al sistema tolemaico, y por eso se me dificulta tanto entender lo que quiere decir sir Karl cuando a ese sistema, o a cualquier otra teoría anacrónica, le llama error" (Kuhn 1982, p. 203).

Aterrizando esta reflexión en una perspectiva hermenéutica, podemos apreciar mejor la naturaleza de la negatividad implícita en un cambio histórico:

Cuando tenemos una experiencia con un objeto, esto quiere decir que hasta entonces no habíamos visto correctamente las cosas y que finalmente en ese momento nos hemos dado cuenta de cómo son. La negatividad de la experiencia posee, en consecuencia, un particular sentido productivo. No es simplemente un engaño que se vuelve visible y, por ende, una corrección, sino que lo que se adquiere es un saber abarcador. (Ankersmit 2010, p. 288) 
Desde una posición kuhniana, los vínculos con la tradición no son "reflexivos" o "psicológicos". El cambio de paradigma no implica en estricto sentido una "toma de decisión". Es así que el problema con la tradición no estriba en encontrar las razones que componen este "background psicológico" ${ }^{24}$ por el cual un científico acepta o rechaza una hipótesis. En este sentido, como ya ejemplificamos en una cita anterior, el "revelar" popperiano parece querer proporcionarle un sustento "racional" a la actitud psicológica. ${ }^{25}$

Del mismo modo, podemos aducir que la separación que Popper establece entre cultura e historia es artificial, en tanto una separación de tal naturaleza parece seguir dependiendo de las nociones analíticas de exterior e interior; pues mientras la comprensión popperiana intenta posicionarse como una construcción del sentido, Kuhn busca hacer explícito su desarrollo a través del cambio de creencias. Con esto, podemos decir que, desde la postura del racionalismo crítico, la historia no cuenta con un papel activo en el cambio científico, sino que se antepone como el límite al cambio, franqueable únicamente por la actitud crítica, a decir de Popper, el exclusivo "detonante" del cambio. La historia se configura así como una tendencia al límite de lo que se puede esperar, creer o "comprender" en general, sin llegar a sostener un carácter "racional" o verse constituida por algún orden. De tal modo, por ejemplo, desde el punto de vista popperiano, la reconstrucción del contexto y su comprensión implican dos procesos únicamente unidos por la critica.

La recuperación del valor de la movilidad de "lo interno" resulta un elemento de importancia en la filosofía kuhniana del cambio en tanto que esta movilidad es a final de cuentas una expresión de la propia incompletud de lo transmitido, entendida esta a su vez como una posibilidad del cambio y no como una limitación:

Debemos reconocer lo muy limitado que puede ser un paradigma en alcance y precisión en el momento de su primera aparición. Los paradigmas obtienen su status como tales, debido a que tienen más éxito que sus competidores para resolver unos cuantos problemas que el grupo de profesionales ha llegado a reconocer como agudos. Sin embargo, el tener más éxito no quiere decir que tenga un éxito completo en la resolución de un problema determinado o que dé resultados suficientemente satisfactorios con un número considerable de problemas. Recordemos que la ciencia normal consiste en gran parte en los intentos de satisfacer la promesa de resolución de tales problemas. (Kuhn 1971, p. 52)

Esta posibilidad-promesa, se asienta en la propia naturaleza inacabada de los paradigmas. Ya hemos reconocido aquí a la historicidad a través del carácter circular que le imprime movimiento a los "saberes", sin embargo, la "necesidad" de esta circularidad se expresa en otro atributo de lo histórico, a decir, en el mismo carácter inacabado o incompleto de los paradigmas:

Como repetidamente hemos subrayado con anterioridad, ninguna teoría resuelve nunca todos los problemas a que en un momento dado se enfrenta, ni es frecuente que las soluciones ya alcanzadas sean perfectas. Al contrario, es justamente lo incompleto y lo imperfecto del ajuste entre la teoría y los datos existentes lo que, en cualquier momento, define muchos de los enigmas que caracterizan a la ciencia normal. (Kuhn 1971, p. 228)

De nueva cuenta, frente al análisis popperiano, podemos decir que en la filosofía del cambio científico de Kuhn, se explicita la movilidad de la ciencia, ahondando en la particularidad de lo incompleto de los paradigmas, es decir, en lo que el paradigma no ha podido resolver como resultado de su misma naturaleza histórica:

Reconociendo que un descubrimiento histórico rara vez es atribuido a su autor en los textos posteriores -los objetivos pedagógicos transforman inevitablemente su narración-, el historiador debe preguntarse

\footnotetext{
24 “Debe comprenderse claramente que sólo hay dos actitudes posibles hacia la tradición. Una es aceptar una tradición sin crítica, a menudo hasta sin ser consciente de ella. En muchos casos, no podemos evitarlo, pues con frecuencia no nos damos cuenta, simplemente, de que estamos frente a una tradición. Si llevo mi reloj en la muñeca izquierda, puedo no ser consciente de esto aceptando una tradición. Todos los días hacemos cientos de cosas bajo la influencia de tradiciones de las que no somos conscientes. Luego, si no sabemos que estamos actuando por influencia de una tradición, no podemos evitar el aceptar la tradición de manera acrítica" (Popper 1983, p. 158).

${ }^{25}$ Kuhn se previene precisamente de esta consideración frente a la interpretación del propio Popper sobre la "fuente de inspiración del individuo" (Kuhn 1982, p. 315).
} 
qué es lo que el sujeto pensaba haber descubierto y en qué se basó para hacer el descubrimiento. Y en este proceso, de reconstrucción, el historiador debe poner especial atención a los aparentes errores del sujeto, no por el gusto de encontrarlos, sino porque ellos revelarán mucho más de la mentalidad activa de su personaje, que los pasajes en los cuales un científico parece registrar un resultado o un argumento que la ciencia moderna retiene todavía. (Kuhn 1982, p. 134)

Desde la misma incompletud de los paradigmas es que se hace necesaria la "irrupción" de la interpretación y se posiciona a la comprensión como la figura que posibilita la pertenencia a un paradigma. Desde este punto de vista, es decir, desde la noción de incompletud, es que puede concebirse la condición histórica de la interpretación como la: "inacababilidad [que] no es defecto de la reflexión sino que está en la esencia misma del ser histórico que somos. Ser histórico quiere decir no agotarse nunca en el saberse” (Gadamer 1999, p. 372).

\section{El fenómeno hermenéutico de la irreductibilidad}

La introducción de los planteamientos propios de la hermenéutica filosófica de H.G. Gadamer resulta sugerente para explicitar la naturaleza histórica de los paradigmas, en especial, con base en la noción de historia efectual que implica directamente a las cuestiones de la relación pasado-presente. Desde dicha concepción, toda relación se describe en términos de una "composición" que involucra centralmente la "irreductibilidad" de los elementos constitutivos en la relación. Bajo esta consideración, no cabe pensar en la reducción o eliminación de ningún elemento de la relación, es decir, en la anulación de la relación misma, lo que invita en este sentido concebir tanto al pasado como el presente como "partes" constitutivas del devenir de la ciencia.

Dentro de la hermenéutica filosófica de Gadamer, la problemática se encuentra directamente entrelazada con la concepción de horizonte, es decir, la noción que involucra a la interpretación como una situación histórica, la historicidad:

La conciencia de la historia efectual es en primer lugar conciencia de la situación hermenéutica. Sin embargo, el hacerse consciente de una situación es una tarea que en cada caso reviste una dificultad propia. El concepto de situación se caracteriza porque uno no se encuentra frente a ella y por lo tanto no puede tener un saber objetivo de ella. Se está en ella, uno se encuentra siempre en una situación cuya iluminación es una tarea a la que nunca se puede dar cumplimiento por entero. Y esto vale también [...] para la situación en la que nos encontramos frente a la tradición que queremos comprender. Tampoco se puede llevar a cabo por completo la iluminación de esta situación. (Gadamer 1999, p. 372)

Con lo anterior podemos decir que es precisamente la incompletud de los elementos que conforman a la estructura, la que fundamenta la condición de apertura, el ser histórico de los procesos de cambio. En este sentido, la "confluencia" entre pasado y presente queda explicada en el marco de la naturaleza dinámica de ambos. Así, el pasado se "convierte" en algo que se hereda y el presente en algo cuya manifestación implícita, involucra el recibimiento o la llegada de lo transmitido y por lo tanto su transformación.

A diferencia de los enfoques "internalistas tradicionales" de la historia, la atención que presta Kuhn al análisis histórico de la ciencia no se centra en las particularidades del contexto en el que un concepto adquiere su sentido, sino en la manera en que este adquiere su sentido a raíz de la apertura de los “contextos". Así pues, frente a las propuestas "tradicionales", Kuhn introduce al movimiento, es decir, a la transformación de la estructura como eje analítico de su filosofía, cuestión que como hemos podido ver, resultaba atribuida entonces a factores externos únicamente.

La naturaleza irreductible de lo interno se convierte entonces en lo que posibilita y requiere que la interpretación haga acto de presencia con fines en la comprensión desde posicionamientos inconmensurables. Es así como creemos que se hace eco de la idea de interpretación en los planteamientos kuhnianos, es decir, toda vez que lo "interno" expresa lo irreductible de los paradigmas, en tanto su condición histórica y no así como un trasfondo de significación. En este sentido, la noción de background no 
representa una dificultad en sí para caracterizar lo que desde enfoques disímiles se pudiera confundir con una postura hermenéutica, sin embargo, sí lo que se está asumiendo por la naturaleza de este background al caracterizarle cognitiva, psicológica o epistémicamente. Así, mientras que para Popper lo que se encuentra en el "fondo" es precisamente lo que hace completo y cerrado a un paradigma, y la comprensión no hace más que ir en contra de la incompletud vista como un error del pasado, para Kuhn, lo que la comprensión desde un punto de vista histórico "resuelve", cae en la revelación y en los efectos productivos de la incompletud. Dicho de otra forma, si para Popper la no resolución de un problema representa un error, para Kuhn, representa el punto de partida en un proceso de cambio. De manera pronta salta a la vista que las dos concepciones persiguen vetas analíticas distintas, pues mientras la primera buscar revelar lo que impide el cambio, el objeto de la segunda es precisamente lo que lo posibilita.

En esto consisten entonces las posibilidades de que la dinámica interna de la ciencia pueda ser revelada a través del análisis del cambio. Es aquí donde la concepción histórica del descubrimiento científico cobra toda su relevancia, pues para Kuhn, el descubrimiento implica "sacar a la luz" el movimiento "interno" de la ciencia: "El descubrimiento de un nuevo tipo de fenómeno sigue un proceso complejo que incluye el reconocimiento de que se ha descubierto algo y de qué es ese algo" (Kuhn 1982, p. 195). Es nuestro punto central aquí considerar, que desde este qué y hasta ese algo, no se establece ningún tipo de distancia o vínculo formal, por lo que no se pude demarcar una oposición entre ambos, ni explicitar su relación desde mecanismos lógico-formales como lo pretende por ejemplo el racionalismocritico. Esto quiere decir a su vez, que el descubrimiento científico no se constituye como una unidad espontánea en el mismo sentido en que los fenómenos nuevos no se expresan de manera primaria, sino emergente. Recordemos que "si el descubrimiento del oxígeno se reduce al simple hecho de tener una muestra impura en las manos, entonces el gas habría sido 'descubierto' en la antigüedad por el primer hombre que embotelló aire atmosférico” (Kuhn 1982, p. 193).

El carácter incompleto de los paradigmas, como bien observa Velasco desde la filosofía popperiana, se finca en la constitución particular de su lenguaje y aplicación, temporalmente situados (Velasco 2004, p. 77). No obstante, cabría tomar con cierta consideración las particularidades que Kuhn atribuye a la condición histórica del lenguaje (Kuhn 1989, pp. 100-101). En este sentido, el término irreductible refiere a que, dada su condición histórica, es imposible pensar a los paradigmas como estructuras cerradas:

Ya en el análisis de la hermenéutica romántica hemos podido ver que la comprensión no se basa en un desplazarse al interior del otro, a una participación inmediata de él. Comprender lo que alguien dice es, como ya hemos visto, ponerse de acuerdo en la cosa, no ponerse en el lugar del otro y reproducir sus vivencias [...]. El lenguaje que discurre [...] lleva consigo su propia verdad, esto es, "desvela" y deja aparecer algo. (Gadamer 1999, p. 461)

Desde el punto de vista popperiano, el lenguaje que "está en el fondo" no constituye la dimensión de lo "no expresado", sino que propiamente refiere a lo que es "indebido expresar". La naturaleza de la negación del no bajo tales circunstancias, resulta distinta a lo que desde un punto de vista kuhniano puede significar "no ver". En todo caso, lo que Popper parece buscar es un lenguaje que ha sido ocultado cuando es que debería haberse hecho explícito, donde el sentido de explícito, de antemano, se aduce en disimilitud con el de descubrir. Por este cause, lo que Popper pareciera entender como aquello que se ha cubierto, es lo que ha sido un error en el pasado, abordado de tal manera, en tanto el problema central popperiano circunda lo que no puede ser visto por causa del error, es decir, lo que resulta en sí "evidente" pero se ha errado en ver, planteamiento que parece aproximarnos hacia ciertos resquicios metafísicos que la apelación formalista a la que Popper se adhiere creemos pretende evitar. En este sentido, la concepción popperiana de la "interpretación" parece centrarse en la búsqueda de un lenguaje que ha sido ocultado por suerte de la equivocación, un lenguaje que está disponible ya de antemano terminado y explícitamente accesible. Podemos ver entonces cómo es que, para Popper, la continuidad histórica entre paradigmas no es un atributo que forme en sí parte del cambio, pues bajo su enfoque, las tradiciones deben componer estructuras cerradas para que puedan ser sujetas de crítica: 
Presupone [Popper], como ya indiqué, que una teoría se expresa, o puede expresarse sin distorsión, en forma total que le permite al científico clasificar todo acontecimiento concebible, bien como caso confirmatorio, caso refutatorio o caso improcedente respecto de la teoría. Obviamente, esto es lo que se requiere para que una ley general sea refutable. (Kuhn 1982, p. 307)

Bibliografía

Ankersmit, F. (2010), La experiencia histórica sublime, Ciudad de México: Universidad Iberoamericana.

Aristóteles (2008), Metafísica, Madrid: Alianza Editorial.

Broncano, F. (2006), “Consideraciones epistemológicas acerca del sentido de agencia”, LOGOS Anales del Seminario de Metafisica 39: 7-27.

Foucault, M. (1979), Microfísica del poder, Madrid: La piqueta.

Gadamer, H. G. (1999), Verdad y método, Salamanca: Sígueme.

Galván, M. C. (2009), Nociones hermenéuticas en la filosofía de la ciencia de Thomas S. Kuhn, Tesis doctoral, Ciudad de México: IIF-UNAM.

González de Luna, E. (2004), “El concepto de sentido común en la epistemología de Karl Popper”, Signos Filosóficos 6(11): 131-144.

Gutiérrez, C. (2000), “Círculo o diálogo? El comprender de Heidegger a Gadamer”, ARETÉ Revista de Filosofía 12(1): 133-143.

Jaspers, K. (1933), Ambiente espiritual de nuestro tiempo, Barcelona/Buenos Aires: Editorial Labor.

Kerényi, K. (1999), La religión antigua, Barcelona: Herder.

Kuhn, T. S. (1971), La estructura de las revoluciones científicas, Ciudad de México: F.C.E.

Kuhn, T. S. (1982), La tensión esencial, Ciudad de México: F.C.E.

Kuhn, T. S. (1989), ¿Qué son las revoluciones científicas? y otros ensayos, Barcelona: Paidós/ICE.

Lakatos, I. (1983), La metodología de los programas de investigación científica, Madrid: Alianza Universidad.

Popper, K. (1962), La lógica de la investigación científica, Madrid: Tecnos.

Popper, K. (2006), La sociedad abierta y sus enemigos, Madrid: Paidós.

Popper, K. (1983), Conjeturas y refutaciones, Barcelona: Paidós Básica.

Ricoeur, Paul (2000), La memoria, la historia, el olvido, Buenos Aires: F.C.E.

Schindler, S. (2015), "Scientific Discovery: That-Whats and What-Thats”, ERGO: An Open Access Journal of Philosophy 2(6): 123-148 (accesible en: http://dx.doi.org/10.3998/ergo.12405314.0002.006).

Velasco, A. (2004), "Hacia una filosofía social de la ciencia en Karl. R. Popper”, Signos Filosóficos 6(11): 71-84. 